Chinese Journal of Organic Chemistry

\title{
抗革兰氏阴性菌耐格霉素的形式合成
}

\author{
张世举 ${ }^{a}$ 李晓䑣 ${ }^{b}$ 王 燕*,c 郑宇璁 ${ }^{d}$ 韩世清*,b \\ 郁惠蕾 $d$ 黄莎华 $*, a, c$ \\ ( ${ }^{a}$ 上海应用技术大学化学与环境工程学院 上海 201418) \\ ( ${ }^{b}$ 南京工业大学生物与制药工程学院 南京 211816) \\ ( ${ }^{c}$ 中国科学院上海有机化学研究所 分子合成卓越中心 天然产物有机合成化学重点实验室 上海 200032) \\ ( $d$ 华东理工大学 生物反应器工程国家重点实验室 上海生物制造技术协同创新中心 上海 200237)
}

\begin{abstract}
摘要 耐格霉素是具有抗革兰氏阴性菌活性的天然产物. 以廉价易得的 3-羰基-4-氯丁酸乙酯为原料, 以八步 $29 \%$ 的总 收率实现了耐格霉素的形式合成. 该工作改进了文献的合成路线, 利用生物催化不对称还原高立体选择性引入 C-5 位 的手性羟基，并将危险的叠氮引入反应放在合成后期，降低合成路线的操作风险. 分子中 C-3 位的仲碳胺基手性中心 通过 Ellamn 试剂介导的不对称 Mannich 反应构建. 该路线易于放大，有望为构建耐格霉素类似物分子库以及高通量药 物篮选奠定基础.
\end{abstract}

关键词＼cjkstart耐格霉素; Mannich 反应; 革兰氏阴性菌; 形式合成

\section{Formal Synthesis of Gram-Negative Antibiotic Negamycin}

$$
\text { Zhang, Shiju }{ }^{a} \quad \text { Li, Xiaotong }{ }^{b} \quad \text { Wang, Yan*,c }{ }^{*} \text { Zheng, Yucong }{ }^{d}
$$

Han, Shiqing $^{*, b} \quad$ Yu, Huilei $^{d} \quad$ Huang, Shahua $^{*, a, c}$

$\left({ }^{a}\right.$ School of Chemical and Environmental Engineering, Shanghai Institute of Technology, Shanghai 201418)

$\left({ }^{b}\right.$ College of Biotechnology and Pharmaceutical Engineering, Nanjing Tech University, Nanjing 211816)

( ${ }^{c}$ Key Laboratory of Synthetic Chemistry of Natural Substances, Center for Excellence in Molecular Synthesis, Shanghai Institute of Organic Chemistry, Chinese Academy of Sciences, Shanghai 200032)

( ${ }^{d}$ State Key Laboratory of Bioreactor Engineering, Shanghai Collaborative Innovation, Center for Biomanufacturing, East China University of Science and Technology, Shanghai 200237)

\begin{abstract}
Negamycin is a potent gram-negative antibiotic. By using commercial available ethyl 4-chlorobutyrate as starting material, the formal synthesis of negamycin was achieved within 8 steps and $29 \%$ overall yield. This modified synthetic route features in-situ enzymatic promoted asmmetric reduction reaction to introduce chiral hydroxy group at C-5, a late-stage azidination at $\mathrm{C}-6$ to avoid the introduction of explosive azide group in the early stage in previous syntheses. The C-3 aza-chiral center was constructed via Ellman reagent-based asymmetric Mannich reaction. This efficient route is scalable and suitable to establish a library of negamycin analogues for future high-throughput screening.
\end{abstract}

Keywords negamycin; Mannich reaction; gram-negative pathogens; formal synthesis

抗生素通过抑制致病菌的生长，使得许多感染性疾 病得到有效治疗, 延长了人类的寿命, 但是同时也促进 了细菌的进化, 导致耐药菌株的产生 ${ }^{[1]}$. 目前, 细菌抗 药性成为威胁人类健康的严重问题，且预计到 2050 年, 每年由于细菌抗药性问题引起的人死亡数将达到上千
万并将耗费 100 万亿美元 ${ }^{[2]}$. 而在众多抗药性细菌中, 由于革兰氏阴性菌的细胞壁上包裹一层具有保护作用 的外膜, 从而阻滞一些抗生素、染料等进入细胞, 且其 引起的抗药性最为严重. 过去半个世纪，临床上没有引 入有效的新类型抗生素. 2017 年世界卫生组织宣布，寻

\footnotetext{
* Corresponding authors. E-mail: wangyan@sippe.ac.cn; hanshiqing@njtech.edu.cn; shahua@sit.edu.cn Received August 16, 2019; revised October 10, 2019; published online October 25, 2019.

Project supported by the National Natural Science Foundation of China (No. 21402121) and the Shanghai Science and Technology Commission for the Shanghai Sailing Program (No. 16YF1414400).

国家自然科学基金(No. 21402121)和上海市杨帆计划(No. 16YF1414400)资助项目.
} 
找新的抗生素抑制革兰氏阴性菌感染是当前最为迫切 的任务之一 ${ }^{[3]}$, 而天然产物结构丰富多样, 一直以来是 人们发现通过新作用机制抵御耐药细菌的新药物分子 的重要灵感源泉 ${ }^{[2]}$.

1970 年, Umezawa 小组 ${ }^{[4]}$ 从绛红褐链霉菌(Streptomyces purpeofuscus) 的培养基中分离得到一种具有抗革 兰氏阴性菌包括铜绿假单胞菌( P. aeruginosa) 以及克雷 白氏肺炎杆菌 (K. pneumoniae) 的抗生素-耐格霉素 (negamycin, 或称负霉素)(图 1). 进一步的研究发现表 明, 耐格霉素通过影响蛋白 RNA 的翻译启动、链增长 以及终止引起密码子错译进而抑制细菌蛋白的合成, 达 到抑制细菌生长的作用 ${ }^{[5]}$. 此外, 耐格霉素具有对提前 终止密码子(PTCs)通读的生理活性, 在杜氏肌营养不良 症(DMD)小鼠模型显示出抑制抗肌营养不良蛋白无义 基因突变的作用 ${ }^{[6]}$. 无义基因突变可导致基因解读提前 终止，从而影响蛋白的合成. 耐格霉素对无义突变基因 的通读作用可以促使蛋白完整地表达, 是杜氏肌营养不 良症的潜在对症药物分子.

正因为耐格霉素是一个结构简单且具有良好的广 谱抗革兰氏阴性菌活性的天然产物, 其结构一经报道就 引起了合成化学家和药物化学家极大的研究兴趣 ${ }^{[7]}$. 1972 年, Shibahara 小组以 $D$-半乳糖醛酸为原料, 首次完 成了耐格霉素的不对称合成, 并确定了其绝对构型 ${ }^{[7 a]}$, 随后有二十多条合成路线相继报道. 在众多的合成路线 中, 以 Oliver 小组 ${ }^{[7 \mathrm{~d}]}$ 的八步合成法路线最短, 他们以手 性的伯醇衍生物为原料, 利用手性辅基介导的不对称 Mannich 反应构建手性 C3 中心. 但是由于其 Mannich 反应选择性低 $(d r \approx 5 / 1)$ 以及路线初期使用易爆物叠氮 化钠，限制了该路线的可操作性和实验室放大. 与此同 时, 有些小组进行了该化合物的结构类似物的构效关系 研究, 以篎选出具有更优良抗菌活性的先导化合物 ${ }^{[8]}$. 为了进一步提高合成效率、促进该类型化合物活性分子 库的快速建立, 便于药物分子的高通量筷选, 本文在已 有文献的基础上 ${ }^{[7 \mathrm{~d}]}$, 设计改进合成步骤, 以便发展高效 的合成策略，实现高对映体纯的耐格霉素的合成.<smiles>CN(CC(=O)O)NC(=O)CC(N)C[C@@H](O)CN</smiles>

(+)-negamycin (1)

图 1 耐格霉素的结构

Figure 1 Structure of $(+)$-negamycin

\section{1 结果与讨论}

虽然耐格霉素结构并不复杂，但是众多的合成路线 由于操作繁琐，不易放大，而且难以获得高光学纯度的 目标化合物. 其中通过还原叠氮引入 C-6 位的氨基是文 献合成路线中常用的构建氨基官能团的方法之一，很多 合成路线在全合成的早期就引入叠氮官能团. 为了积累 后续中间体，沿用相似的策略，放大合成时就会在操作 上带来一定的安全隐患. 为了减少易致爆叠氮原料(如 $\mathrm{NaN}_{3}$ ) 的使用，本策略采用后期引入叠氮官能团的方法 完成 C-6 位氨基的引入(Scheme 1). 而 C-1 位的酰胺肼 片段则由羧酸 3 与文献已知的肼衍生物 $\mathbf{4}^{[9]}$ 通过酰胺缩 合方式构建. 关键的合成中间体 3 可以通过不对称的 Mannich 反应得到，通过底物亚胺上的手性亚砜(Ellman 试剂 $)^{[10]}$ 取代基控制反应的立体选择性，从而建立 C-3 位的手性中心. 亚胺中间体 $\mathbf{5}$ 可由生物酶催化还原大量 获得的手性化合物 $\mathbf{6}^{[11,8 \mathrm{f}]}$ 通过简单的还原和氨基缩合 来制备。

尽管利用过渡金属催化不对称还原是很成熟的方 法 $^{[12]}$, 生物催化由于其条件温和及高度立体专一性更 符合绿色化学的要求, 并在一些药物合成中成功地应 用 ${ }^{[13]}$. 例如，酶促不对称还原 3-羰基-4-氯丁酸乙酯已成 功应用于他汀类药物侧链的工业生产 ${ }^{[14]}$. 我们受此启 发, 将来源于平滑假丝酵母(Candida glabrata)的具备相 反立体选择性的大肠杆菌重组酮还原酶 CgKR1 作为催 化剂 ${ }^{[11]}$, 耦合来源于巨大芽孢杆菌(Bacillus megaterium) 葡萄糖脱氢酶 $B m \mathrm{GDH}$ 进行 NADPH 辅酶再生, 在中性 条件下避免了低温反应, 以及硼烷还原法后处理过程中 底物酯基水解的问题. 利用该方法，可大量制得手性着弪

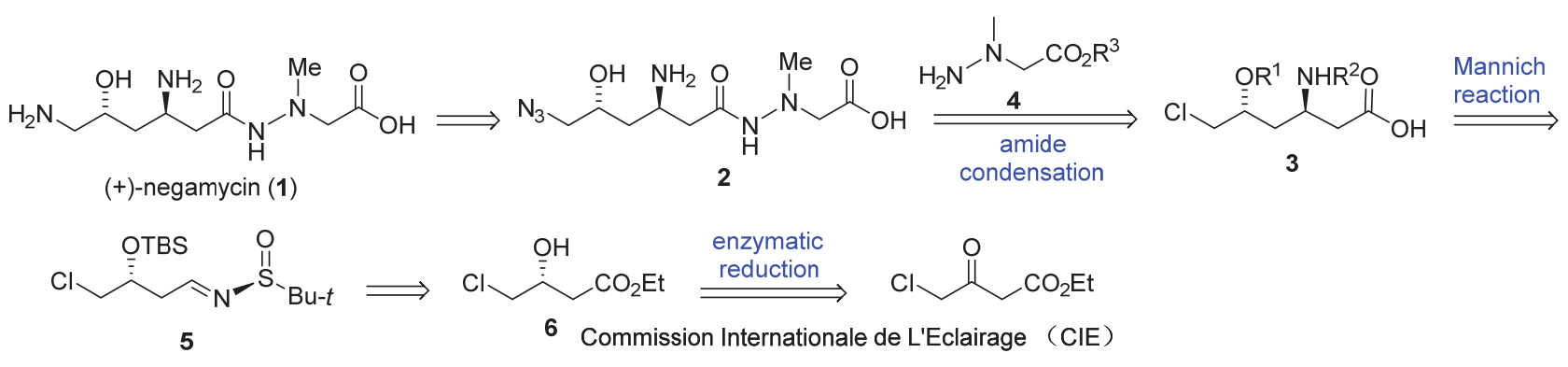

图式 1 耐格霉素的逆合成分析

Scheme 1 Retro-synthetic analysis of (+)-negamycin 
基化合物 $6(96 \% e e)$. 以 6 原料, 在碱性条件下利用二甲 基叔丁基硅基氯( $\mathrm{TBSCl}$ )保护羟基，随后在低温条件下， 二异丙基铝化氢(DIBALH)选择性地将酯基还原为醛基 (Scheme 2). 所得丁醛衍生物在四乙氧基钣作用下与 $(R)-(-)$ - 叔丁基亚磺酰胺(Ellman 试剂)发生缩合反 应 $^{[15]}$, 以 $84 \%$ 的收率制备得到 Mannich 反应前体亚胺衍 生物 5.

在获得亚胺衍生物 $\mathbf{5}$ 后, 首先利用在二异丙胺基锂 (LDA)作用下, 对烯醇锂盐与亚胺的 Mannich 反应进行 尝试, 并未检测到所需的加成产物生成. 分析可能是烯 醇锂盐与亚胺衍生物的酯基发生了 Claisen 缩合. 根据 文献报道, 烯醇锂盐能与三异丙氧基氯化钛发生转金属 化反应生成烯醇钛盐, 该钛盐不仅能促进 Mannich 反应 的进行, 并能提高该反应的立体选择性 ${ }^{[13]}$. 随后 Ellman 等 ${ }^{[16]}$ 发展了烯醇钛盐参与的 Mannich 反应条件, 将烯醇 锂盐与三异丙氧基氯化钛在低温下搅拌 $40 \mathrm{~min}$, 原位制
备烯醇钛盐，随后滴入亚胺衍生物 $\mathbf{5}$, 保持低温反应 $3 \mathrm{~h}$, 所得产物分离鉴定，得到了单一化合物 13 (Scheme 3). 我们推测烯醇钛盐首先与亚胺发生 Mannich 反应生成化 合物 9, 该化合物进一步与体系中过量的烯醇钣盐发生 Claisen 缩合转化为 13. 进一步控制加成反应 $1 \mathrm{~h}$, 以 $84 \%$ 的分离产率得到单一的非对映异构体 $\mathbf{9}$, 且未发现 Claisen 缩合产物 13 的生成. 在该反应中, 烯醇针盐与 亚胺形成椅式六元环过渡态，由于亚砜上叔丁基的立体 位阻效应, 使得过渡态 TS1 中相邻亚胺的侧链 $(\mathrm{R})$ 也处 于直立键, 因此亚胺的 $S i$ 面接受亲核试剂进攻, 产生单 一的手性胺衍生物. 显然, 六元环过渡态中, 两个相邻 大位阻取代基均处于平伏键反而是不利的，因此过渡态 TS2 不是优势过渡态. 而文献中类似反应过程只取得 $5 / 1$ 的非对映选择性和 $41 \%$ 的分离收率 ${ }^{[7 d]}$, 可见远端的 官能团(C-6 取代基)可能对反应的控制产生一定的影响.<smiles>CCOC(=O)CC(=O)CCl</smiles>

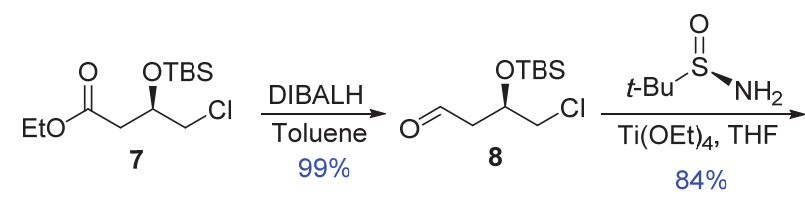<smiles>CC(C)(C)S(=O)/N=C/CC([O+])CCl</smiles>

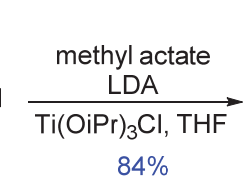<smiles>CC(=O)CC(CC(CCl)C(C)(C)C)NS(=O)C(C)(C)C</smiles><smiles>CC(C)(C)[13C](=O)N[C@@H](CC(=O)O)C[C@@H](CCl)O[Mg]</smiles><smiles>CCOC(=O)CN([NH3+])[N+](=O)[O-]</smiles>
10<smiles>CCOC(=O)CN(C)NC(=O)C[C@H](CC(CCl)O[Sn](F)(F)F)NS(=O)CC(C)C</smiles>
11<smiles>CCCCOC(CCC)C[C@H](CC(=O)NN(C)CC(=O)OCC)NS(=O)CC(C)(C)C</smiles>

12<smiles>CN(CC(=O)O)NC(=O)C[C@H](N)C[C@H](O)CN</smiles>

(+)-negamycin (1)

图式 2 耐格霉素的形式合成路线

Scheme 2 Formal synthesis route of ( + -negamycin

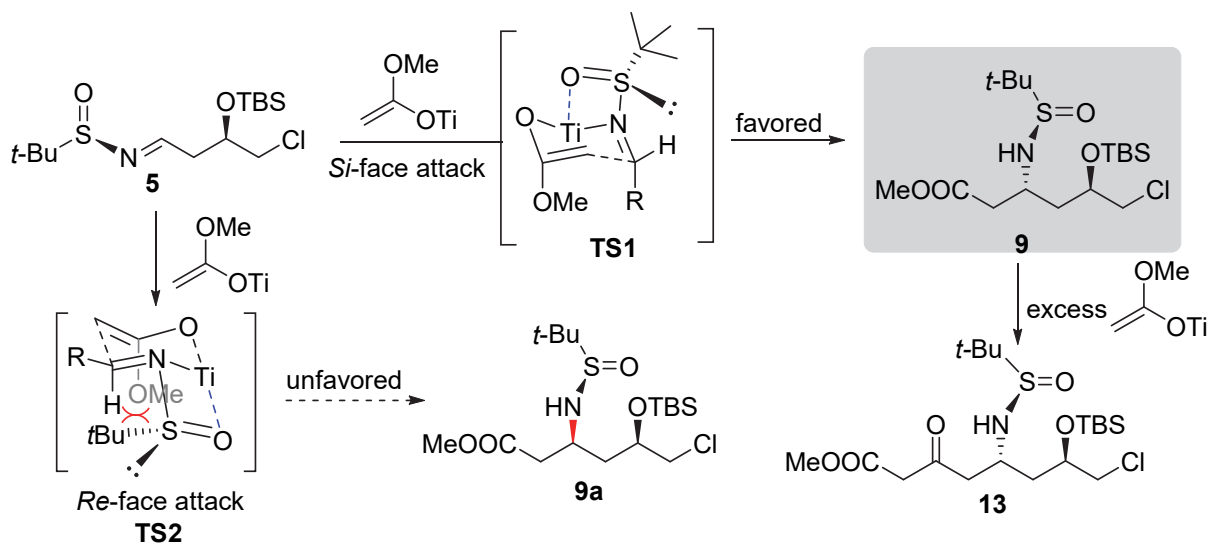

图式 3 Mannich 反应可能过渡态

Scheme 3 Proposed transition state of Mannich reaction 
在碱性条件下, Mannich 产物的甲酯发生水解反应 转化为相应的羧酸, 该羧酸在缩合条件下与文献已知的 肼衍生物 4 发生酰胺缩合反应, 以两步 83\%总收率得到 化合物 11, 完成了所有碳骨架的建立. 相对于目标产 物, 需要在 C-6 位引入氨基, 本路线仍采用经典的叠氮 还原法构建氨基. 在加热条件下, 化合物 11 与叠氮化钠 发生 $\mathrm{S}_{\mathrm{N}} 2$ 取代反应，以 $66 \%$ 的收率制备得到文献已知关 键中间体 $12^{[7 \mathrm{dd}]}$. 化合物 12 可通过简单的氢化、酸脱除 保护两步转化即可得到天然产物耐格霉素.

\section{2 结论}

从商品化的的 3-羰基-4-氯丁酸乙酯出发, 首先利 用双酶耦法方便地构建出 C-5 位的手性羟基, 进而利用 Ellman 辅基控制的不对称 Mannich 反应构建 C-3 位的手 性中心, 并在合成后期引入具有潜在制备风险的叠氮官 能团, 以 8 步 $29 \%$ 的总收率完成了耐格霉素的形式合成. 该合成策略为快速建立耐格霉素类似物分子库提供了 一种新的合成路线, 有助于开展类似物的合成, 推动构 效关系研究, 加快寻找高活性抗菌药物的步伐.

\section{3 实验部分}

\section{1 仪器与试剂}

核磁数据用 Bruker AM-400 (400 MHz)核磁共振仪 测定的; 红外光谱用 Digital FT-IRspectrometer 或 Bruker-Tensor 27 测定; 质谱用 Shimadzu LCMS-2010EV (ESI) mass spectrometer 或 Agilent G6100 LC/MSD(ESI) 质谱仪测定; 高分辨质谱采用 Bruker Daltonics, Inc. APEXIII 7.0 TESLA FTMS (ESI) 质谱仪测定; 比旋光值 通过 JASCO P-1030 polarimeter 测定.

所有试剂除了特殊说明都是直接使用; 四氢呋喃与 甲苯都是经金属钠和二苯甲酮回流至蓝色后蒸出; 二氯 甲烷是由氢化钻干燥新蒸.

\section{2 实验方法}

\subsection{1 生物催化制备 $(R)$-4-氯-3-羟基丁酸乙酯(6)}

含有 $5 \mathrm{~g}$ 重组 pET-28(a)+-CgKR1 大肠杆菌湿细胞 的细胞裂解液 $(47 \mathrm{~mL}$, 总活力 $3000 \mathrm{U}$, 细胞悬浮于 500 $\mathrm{mmol} \cdot \mathrm{L}^{-1}$ 磷酸钾缓冲液, $\mathrm{pH} 6.0$, 冰浴超声破碎) 与无水 葡萄糖 $(27 \mathrm{~g}), B m \mathrm{GDH}$ (葡萄糖脱氢酶) 冻干酶粉 $(1.5 \mathrm{~g}$, 总活力 $4500 \mathrm{U}$ ), 底物 3-羰基-4-氯丁酸乙酷酮 $(15 \mathrm{~g}, 91.5$ $\mathrm{mmol}$ )及乙醇 $(3.0 \mathrm{~mL})$ 在 $25{ }^{\circ} \mathrm{C}$ 摚拌 $36 \mathrm{~h}$. 期间通过 $\mathrm{pH}$ 滴定仪以碳酸钠水溶液恒定 $\mathrm{pH}$ 6.0. 反应结束后体系加 入氯化钠 $(27 \mathrm{~g})$, 以乙酸乙酯萃取, 以离心机分相. 合并 有机相, 无水硫酸镁干燥, 水泵真空除溶剂, 柱层析纯 化 $[V$ (乙酸乙酯 $) / V($ 石油醚 $)=1 / 6$ ] 得淡黄色油状液体 $\mathbf{6}^{[11]}$ $13.5 \mathrm{~g}$, 产率 $89 \%, 96 \%$ ee. $[\alpha]_{\mathrm{D}}^{25}-21.4\left(c 1.0 \mathrm{CHCl}_{3}\right) ;{ }^{1} \mathrm{H}$
NMR (400 MHz, $\left.\mathrm{CDCl}_{3}\right) \delta: 4.23 \sim 4.30(\mathrm{~m}, 1 \mathrm{H}), 4.19$ (q, $J=7.1 \mathrm{~Hz}, 2 \mathrm{H}), 3.57 \sim 3.66(\mathrm{~m}, 2 \mathrm{H}), 3.21(\mathrm{~d}, J=5.2 \mathrm{~Hz}$, $1 \mathrm{H}), 2.57 \sim 2.70(\mathrm{~m}, 2 \mathrm{H}), 1.29(\mathrm{t}, J=7.2 \mathrm{~Hz}, 3 \mathrm{H})$.

3.2.2 (R)-3-叔丁基二甲基硅基氧基-4-氯丁酸乙酯 (7)的制备

在 $100 \mathrm{~mL}$ 茄形瓶中加入咪唑 $(6.80 \mathrm{~g}, 100 \mathrm{mmol})$, 氮气保护下溶于 $20 \mathrm{~mL}$ 超干 $N, N$-二甲基甲酰胺(DMF) 中, 用冰水浴冷却至 $0{ }^{\circ} \mathrm{C}$ 后, 滴加仲醇化合物 $6(3.3 \mathrm{~g}$, $20 \mathrm{mmol})$, 并加入 TBSCl (3.9 g, $26 \mathrm{mmol})$, 加料完成后 常温下摚拌反应 $24 \mathrm{~h}$. 反应结束后加入饱和碳酸氢钠水 溶液 $(5 \mathrm{~mL})$ 淬灭反应, 加入 $20 \mathrm{~mL}$ 水充分搅拌, 分离有 机相, 用乙醚 $(30 \mathrm{~mL} \times 3)$ 萃取水相, 合并有机相, 用饱 和氯化钠水溶液洗涤, 无水硫酸钠干燥, 过滤, 浓缩, 柱层析纯化 $[V($ 乙酸乙酯 $) / V($ 石油醚 $)=1 / 100]$ 得到黄色油 状物 ${ }^{[17]} 4.82 \mathrm{~g}$, 产率 $85 \%$. [ $\left.\alpha\right]_{\mathrm{D}}^{25}+18.0\left(c 1.03, \mathrm{CHCl}_{3}\right)$; ${ }^{1} \mathrm{H}$ NMR $\left(400 \mathrm{MHz}, \mathrm{CDCl}_{3}\right) \delta: 4.34 \sim 4.28(\mathrm{~m}, 1 \mathrm{H})$, $4.20 \sim 4.08(\mathrm{~m}, 2 \mathrm{H}), 3.55 \sim 3.47(\mathrm{~m}, 2 \mathrm{H}), 2.68(\mathrm{dd}, \quad J=$ 15.2, $4.8 \mathrm{~Hz}, 1 \mathrm{H}), 2.52(\mathrm{dd}, J=15.2,7.2 \mathrm{~Hz}, 1 \mathrm{H}), 1.27(\mathrm{t}$, $J=7.2 \mathrm{~Hz}, 3 \mathrm{H}$ ), 0.87 (s, 9H), 0.09 (d, $J=15.6 \mathrm{~Hz}, 6 \mathrm{H})$.

$3.2 .3(R)-3$ - 叔丁基二甲基硅基氧基-4-氯丁醛 $(8)$ 的 制备

在氮气气氛下, 将化合物 $7(11.6 \mathrm{mmol}, 3.28 \mathrm{~g})$ 置于 $250 \mathrm{~mL}$ 茄形瓶中, 用 $100 \mathrm{~mL}$ 新蒸甲苯溶解, 并将反应 温度降至 $-78{ }^{\circ} \mathrm{C}$, 待温度稳定后滴加二异丁基氢化铝 $\left(1.5 \mathrm{~mol} \cdot \mathrm{L}^{-1}\right.$ 甲苯溶液, $\left.8.5 \mathrm{~mL}, 12.8 \mathrm{mmol}\right)$, 反应 $1 \mathrm{~h}$ 后, 加入无水甲醇 $(3 \mathrm{~mL})$ 淬灭反应. 恢复至室温, 向体系中 加入饱和酒石酸钠钾溶液 $(50 \mathrm{~mL})$, 充分摚拌 $2 \mathrm{~h}$ 至体系 澄清. 分离有机相，水相用乙醚 $(30 \mathrm{~mL} \times 3)$ 萃取，合并 有机相, 用饱和氯化钠水溶液洗涤, 无水硫酸钠干燥, 过滤, 浓缩, 柱层析纯化 $[V($ 乙酸乙酯 $) / V$ (石油醚 $)=$ $1 / 40$ ], 得到黄色油状化合物 $\mathbf{8}^{[17]} 2.73 \mathrm{~g}$, 产率 $99 \%$. $[\alpha]_{\mathrm{D}}^{23}+17.1\left(c 1.01, \mathrm{CHCl}_{3}\right) ;{ }^{1} \mathrm{H} \mathrm{NMR}\left(400 \mathrm{MHz}, \mathrm{CDCl}_{3}\right)$ $\delta: 9.80(\mathrm{t}, J=1.6 \mathrm{~Hz}, 1 \mathrm{H}), 4.41 \sim 4.37(\mathrm{~m}, 1 \mathrm{H}), 3.54(\mathrm{dd}$, $J=11.2,4.8 \mathrm{~Hz}, 1 \mathrm{H}), 3.47(\mathrm{dd}, J=10.8,6.4 \mathrm{~Hz}, 1 \mathrm{H})$, $2.81 \sim 2.65(\mathrm{~m}, 2 \mathrm{H}), 0.87$ (s, 9H), $0.10(\mathrm{~d}, J=14.4 \mathrm{~Hz}, 6 \mathrm{H})$. 3.2.4 (R)-( $N$ - $(R)$-叔丁基亚磺酰基)-3-叔丁基二甲基 硅基氧基-4-氯-1-丁亚胺 $(5)$ 制备

在 $100 \mathrm{~mL}$ 圆底烧瓶中加入 $(R)-(+)$ 叔丁基亚磺酰胺 $(1.7 \mathrm{~g}, 14.2 \mathrm{mmol})$, 氮气保护下溶于 $40 \mathrm{~mL}$ 新蒸四氢呋 喃中, 置于冰水浴中冷却至 $0{ }^{\circ} \mathrm{C}$ 后，依次滴加化合物 8 $(2.8 \mathrm{~g}, 11.8 \mathrm{mmol})$ 的四氢呋喃(THF) $(12 \mathrm{~mL})$ 的溶液, 四 乙氧基钛 $(5 \mathrm{~mL}, 23.6 \mathrm{mmol})$, 加料完成后恢复至室温, 加热回流反应 $1 \mathrm{~h}$. 停止加热并冷却至室温, 加入饱和 氯化钠水溶液 $(5 \mathrm{~mL})$ 淬灭反应, 硅藻土过滤, 用乙醚洗 涤滤饼, 将滤液浓缩以除去大部分的 THF. 向所得的残 
余物中加入乙醚稀释, 分离有机相, 水相用乙醚(20 $\mathrm{mL} \times 3)$ 萃取, 合并有机相, 用无水硫酸钠干燥, 过滤, 浓缩后用柱层析纯化 $[V($ 乙酸乙酯 $) / V($ 石油醚 $)=1 / 20]$ 得 到黄色油状化合物 $53.4 \mathrm{~g}$, 产率 84\%. [ $\alpha]_{\mathrm{D}}^{25}-117.0(\mathrm{c}$ $\left.1.19, \mathrm{CH}_{2} \mathrm{Cl}_{2}\right) ;{ }^{1} \mathrm{H}$ NMR $\left(400 \mathrm{MHz}, \mathrm{CDCl}_{3}\right) \delta: 8.12$ (t, $J=$ $4.8 \mathrm{~Hz}, 1 \mathrm{H}), 4.30 \sim 4.24(\mathrm{~m}, 1 \mathrm{H}), 3.55$ (dd, $J=10.8,4.8$ $\mathrm{Hz}, 1 \mathrm{H}), 3.50(\mathrm{dd}, J=11.2,6.4 \mathrm{~Hz}, 1 \mathrm{H}), 2.90 \sim 2.77(\mathrm{~m}$, 2H), 1.20 (s, 9H), 0.89 (s, 9H), 0.10 (d, $J=8.0 \mathrm{~Hz}, 6 \mathrm{H}$ ); ${ }^{13} \mathrm{C}$ NMR $\left(100 \mathrm{MHz}, \mathrm{CDCl}_{3}\right) \delta: 166.7,70.1,56.9,48.0$, 41.2, 25.8, 22.5, 18.1, -4.5, -4.6; IR (KBr) v: 2956, 2929, 2898, 2858, 1737, 1622, 1089, $838 \mathrm{~cm}^{-1}$; ESI-LR $m / z: 340.2[\mathrm{M}+\mathrm{H}]^{+}$; HRMS (ESI) calcd for $\mathrm{C}_{14} \mathrm{H}_{31} \mathrm{O}_{2} \mathrm{~N}-$ ClSSi $[\mathrm{M}+\mathrm{H}]^{+}$340.1528, found 340.1527.

$3.2 .5(3 R, 5 R)-5$-叔丁基二甲基硅基氧基-3-( $N-(R)$ - 叔 丁基亚磺酰基)氨基-6-氯已酸甲酯(9)的制备

在氮气保护下, 于 $100 \mathrm{~mL}$ 茄形瓶中加入二异丙胺 $(1.5 \mathrm{~mL}, 10.9 \mathrm{mmol}), 10 \mathrm{~mL}$ 新蒸四氢呋喃, 并冷却至 $-78{ }^{\circ} \mathrm{C}$, 缓慢滴入正丁基锂 $\left(2.4 \mathrm{~mol} \cdot \mathrm{L}^{-1}\right.$ 正已烷溶液, $4.8 \mathrm{~mL}, 10.9 \mathrm{mmol})$, 滴加完后缓慢升至 $0{ }^{\circ} \mathrm{C}$ 搅拌 $1 \mathrm{~h}$. 将反应瓶再次冷却至 $-78{ }^{\circ} \mathrm{C}$, 滴入乙酸甲酯 $(778 \mathrm{mg}$, $10.5 \mathrm{mmol})$ 的 THF $(10 \mathrm{~mL})$ 溶液, 滴加完成后保持该温 度摚拌反应 $1 \mathrm{~h}$. 缓慢滴入三异丙氧基氯化钛 $\left(1 \mathrm{~mol} \cdot \mathrm{L}^{-1}\right.$ 正己烷溶液, $21.7 \mathrm{~mL}, 21.7 \mathrm{mmol}$ )后反应 $40 \mathrm{~min}$, 滴入化 合物 5 (1.2 g, $3.5 \mathrm{mmol})$ 的 THF (7 mL) 的溶液. 薄层色谱 (TLC) 跟踪至原料反应完全, 低温下加入无水甲醇 (3 $\mathrm{mL}$ ) 淬灭反应, 恢复至室温, 加 $10 \mathrm{~mL}$ 水充分摚拌后硅 藻土过滤, 乙醚洗涤滤饼, 将滤液浓缩以除去大部分的 THF. 向所得的残余物中加入乙醚稀释, 分离有机相, 水相用乙醚 $(20 \mathrm{~mL} \times 3)$ 萃取, 合并有机相后用无水硫酸 钠干燥, 过滤, 浓缩后柱层析纯化 $[V($ 乙酸乙酯 $) / V($ 石油 醚) $=1 / 4$ ] 得到黄色油状化合物 $91.2 \mathrm{~g}$, 产率 84\%. [ $\alpha]_{\mathrm{D}}^{25}$ - 48.3 (c $0.33, \mathrm{CH}_{2} \mathrm{Cl}_{2}$ ); ${ }^{1} \mathrm{H}$ NMR $\left(400 \mathrm{MHz}, \mathrm{CDCl}_{3}\right.$ ) $\delta$ : $4.34(\mathrm{~d}, J=10.0 \mathrm{~Hz}, 1 \mathrm{H}), 4.02 \sim 4.00(\mathrm{~m}, 1 \mathrm{H}), 3.68(\mathrm{~s}$, $3 \mathrm{H}$ ), 3.46 (dd, $J=10.8,3.6 \mathrm{~Hz}, 1 \mathrm{H}$ ), 3.39 (dd, $J=10.8,6.4$ $\mathrm{Hz}, 1 \mathrm{H}), 2.96$ (dd, $J=16.4,5.6 \mathrm{~Hz}, 1 \mathrm{H}), 2.62(\mathrm{dd}, J=16.8$, $4.4 \mathrm{~Hz}, 1 \mathrm{H}), 1.99 \sim 1.92(\mathrm{~m}, 1 \mathrm{H}), 1.61 \sim 1.54(\mathrm{~m}, 1 \mathrm{H}), 1.23$ (s, 9H), 0.89 (s, 9H), 0.09 (s, 6H); ${ }^{13} \mathrm{C}$ NMR (100 MHz, $\left.\mathrm{CDCl}_{3}\right) \delta: 172.6,69.4,56.3,51.7,51.0,48.9,41.7,41.0$, 25.9, 22.9, 18.1, -4.0, -4.2; IR (KBr) v: 3206, 2955, 2929, 2857, 1740, 1092, 1044, $837 \mathrm{~cm}^{-1}$; ESI-LR $\mathrm{m} / z$ : $414.1[\mathrm{M}+\mathrm{H}]^{+}$; HRMS (ESI) calcd for $\mathrm{C}_{17} \mathrm{H}_{37} \mathrm{O}_{4} \mathrm{NClSSi}$ $[\mathrm{M}+\mathrm{H}]^{+}$414.1896, found 414.1893.

3.2.6 (5R,7R)-7-叔丁基二甲基硅基氧基-5-(N- $(R)$-叔 丁基亚磺酰基)氨基-8-氯-3-羰基已酸甲酯(13)的制备

实验操作同化合物 9 的方法, 反应时间延长至 3.5 $\mathrm{h}$, 分离得到黄色油状物 $13 .{ }^{1} \mathrm{H}$ NMR $\left(400 \mathrm{MHz}, \mathrm{CDCl}_{3}\right)$ $\delta: 4.10(\mathrm{~d}, J=10.5 \mathrm{~Hz}, 1 \mathrm{H}), 4.02 \sim 3.99(\mathrm{~m}, 1 \mathrm{H}), 3.73(\mathrm{~s}$, $3 \mathrm{H}), 3.48 \sim 3.44(\mathrm{~m}, 2 \mathrm{H}), 3.42 \sim 3.37(\mathrm{~m}, 1 \mathrm{H}), 3.18(\mathrm{dd}$, $J=18.2,5.5 \mathrm{~Hz}, 1 \mathrm{H}), 2.94(\mathrm{dd}, J=18.2,3.5 \mathrm{~Hz}, 1 \mathrm{H}), 2.22$ (t, $J=7.6 \mathrm{~Hz}, 1 \mathrm{H}), 2.08 \sim 2.00(\mathrm{~m}, 2 \mathrm{H}), 1.22(\mathrm{~s}, 9 \mathrm{H}), 0.88$ (s, 9H), $0.10(\mathrm{~s}, 6 \mathrm{H})$.

$3.2 .7 \quad(3 R, 5 R)-5$-叔丁基二甲基硅基氧基-3- $(N-(R)$ - 叔 丁基亚磺酰基) 氨基-6-氯已酸甲酸 $(10)$ 的制备

将化合物 9 (2.5 g, $6.0 \mathrm{mmol}$ )置于 $100 \mathrm{~mL}$ 茄形瓶中, 加入 $20 \mathrm{~mL}$ 无水甲醇溶解, 放置于冰水浴中冷却至 $0{ }^{\circ} \mathrm{C}$, 滴加氢氧化锂水溶液 $\left(1 \mathrm{~mol} \cdot \mathrm{L}^{-1}\right.$ 水溶液, $18 \mathrm{~mL}$, $18.0 \mathrm{mmol})$, 滴加完成后恢复至室温反应, TLC 跟踪至 原料反应完全, 反应结束后滴加 $1 \mathrm{~mol} \cdot \mathrm{L}^{-1}$ 盐酸将反应 液将 $\mathrm{pH}$ 调至酸性, 用乙醚 $(40 \mathrm{~mL} \times 4)$ 萃取, 合并有机相 用饱和氯化钠水溶液洗涤, 无水硫酸钠干燥, 过滤, 浓 缩后柱层析纯 $[V(\mathrm{MeOH}) / V(\mathrm{DCM})=1 / 50]$ 得到白色固体 $102.0 \mathrm{~g}$, 产率 $83 \%$. $[\alpha]_{\mathrm{D}}^{25}+7.5$ (c $\left.1.91, \mathrm{CH}_{2} \mathrm{Cl}_{2}\right) ;{ }^{1} \mathrm{H}$ NMR (400 MHz, $\left.\mathrm{CDCl}_{3}\right) \delta: 4.80(\mathrm{~d}, J=9.2 \mathrm{~Hz}, 1 \mathrm{H}), 3.96$ (s, $1 \mathrm{H}), 3.69 \sim 3.63(\mathrm{~m}, 1 \mathrm{H}), 3.48 \sim 3.37(\mathrm{~m}, 2 \mathrm{H}), 3.06 \sim$ $3.01(\mathrm{~m}, 1 \mathrm{H}), 2.52(\mathrm{~d}, J=16.4 \mathrm{~Hz}, 1 \mathrm{H}), 2.16 \sim 2.09(\mathrm{~m}$, $1 \mathrm{H}), 1.64 \sim 1.57(\mathrm{~m}, 1 \mathrm{H}), 1.28(\mathrm{~s}, 9 \mathrm{H}), 0.89(\mathrm{~s}, 9 \mathrm{H}), 0.09$ (s, 6H); ${ }^{13} \mathrm{C}$ NMR (100 MHz, $\left.\mathrm{CDCl}_{3}\right) \delta: 173.8,69.8,57.1$, $51.7,48.9,41.8,40.5,25.9,23.1,18.1,-3.9,-4.2$; IR (KBr) v: 3212, 2956, 2929, 2857, 1718, 1255, 1197, 1094, $837 \mathrm{~cm}^{-1}$; ESI-LR m/z: $398.2[\mathrm{M}-\mathrm{H}]^{-}$; HRMS (ESI) calcd for $\mathrm{C}_{16} \mathrm{H}_{33} \mathrm{O}_{4} \mathrm{NClSSi}[\mathrm{M}-\mathrm{H}]^{-}$398.1594, found 398.1591 .

3.2.8 $N-(3 R, 5 R)-5$-叔丁基二甲基硅基氧基-3- $(N-(R)$ 叔丁基亚磺酰基) 氨基-6-氯已酰胺基- $N$-甲基甘氨酸 叔丁酯(11)的制备

将化合物 $\mathbf{1 0}(563 \mathrm{mg}, 1.4 \mathrm{mmol}), \mathrm{N}$-氨基- $\mathrm{N}$-甲基甘 氨酸叔丁酯对甲磺酸盐 $(515 \mathrm{mg}, 1.55 \mathrm{mmol})$ 和 $\mathrm{HOBt}$ (378 mg, $2.8 \mathrm{mmol})$ 置于 $50 \mathrm{~mL}$ 茄形瓶中, 氮气保护下 加入 $15 \mathrm{~mL}$ 新蒸二氯甲烷溶解, 放置于冰水浴中冷却至 $0{ }^{\circ} \mathrm{C}$ 后, 滴加三乙胺 $(0.4 \mathrm{~mL}, 2.8 \mathrm{mmol})$ 并加入 $\mathrm{EDCI}$ (537 mg, $2.8 \mathrm{mmol})$, 然后恢复至室温搅拌反应. TLC 跟 踪至原料反应完全, 加入 $10 \%$ 柠檬酸水溶液 $(30 \mathrm{~mL})$ 充 分摚拌, 分离有机相, 水相用乙酸乙酯 $(30 \mathrm{~mL} \times 3)$ 萃取, 合并有机相, 依次用饱和碳酸氢钠水溶液和饱和氯化钠 水溶液洗涤, 无水硫酸钠干燥, 浓缩后柱层析纯化 $[V($ 丙酮 $) / V($ 石油醚 $)=1 / 5$ ]得到黄色油状化合物 11762 $\mathrm{mg}$, 产率定量. $[\alpha]_{\mathrm{D}}^{25}-4.7$ ( c $\left.0.85, \mathrm{CH}_{2} \mathrm{Cl}_{2}\right) ;{ }^{1} \mathrm{H} \mathrm{NMR}$ $\left(400 \mathrm{MHz}, \mathrm{CDCl}_{3}\right) \delta: 5.28 \sim 4.96(\mathrm{~m}, 1 \mathrm{H}), 4.02 \sim 3.96(\mathrm{~m}$, $1 \mathrm{H}), 3.67 \sim 3.32(\mathrm{~m}, 5 \mathrm{H}), 3.03 \sim 2.84(\mathrm{~m}, 1 \mathrm{H}), 2.71(\mathrm{~s}$, $1.8 \mathrm{H}$, major isomer), 2.70 (s, $1.2 \mathrm{H}$, minor isomer), $2.23 \sim$ 
$1.90(\mathrm{~m}, 2 \mathrm{H}), 1.67 \sim 1.52(\mathrm{~m}, 1 \mathrm{H}), 1.45(\mathrm{~s}, 9 \mathrm{H}), 1.24(\mathrm{~s}$, $5.7 \mathrm{H}$, major isomer), $1.21(\mathrm{~s}, 3.8 \mathrm{H}$, minor isomer), $0.89(\mathrm{~s}$, $3.6 \mathrm{H}$, minor isomer), $0.87(\mathrm{~s}, 5.4 \mathrm{H}$, major isomer), 0.09 (s, $1.2 \mathrm{H}$, minor isomer) $0.084(\mathrm{~s}, 1.2 \mathrm{H}$, minor isomer $), 0.076$ (s, $1.8 \mathrm{H}$, major isomer), 0.07 ( $\mathrm{s}, 1.8 \mathrm{H}$, major isomer); The ratio of rotamers is $1.5: 1$; IR (KBr) $v: 3236,2956,2929$, 2857, 1744, 1667, 1253, 1225, 1072, $837 \mathrm{~cm}^{-1}$; ESI-LR $m / z: 542.2[\mathrm{M}+\mathrm{H}]^{+}$; HRMS (ESI) calcd for $\mathrm{C}_{23} \mathrm{H}_{48} \mathrm{O}_{5^{-}}$ $\mathrm{N}_{3} \mathrm{ClNaSSi}[\mathrm{M}+\mathrm{Na}]^{+}$564.2665, found 564.2668.

3.2.9 N-(3R,5R)-5-叔丁基二甲基硅基氧基-3- $(N-(R)-$ 叔丁基亚磺酰基) 氨基-6-叠氮已酰胺基- $N$-甲基甘氨 酸叔丁酯(12)的制备

将化合物 11 (279 mg, $0.5 \mathrm{mmol}$ )置于反应管中, 氮 气保护下加入 $3 \mathrm{~mL}$ 超干 DMF 溶解, 加入碘化钾 (83 $\mathrm{mg}, 0.5 \mathrm{mmol})$ 和叠氮化钠 $(98 \mathrm{mg}, 1.5 \mathrm{mmol})$, 体系加热 至 $90{ }^{\circ} \mathrm{C}$ 搅拌反应, ${ }^{1} \mathrm{H}$ NMR 跟踪至原料反应完全, 停止 加热, 冷却至室温, 加入 $5 \mathrm{~mL}$ 水充分搅拌, 分离有机 相, 用乙醚 $(10 \mathrm{~mL} \times 5)$ 萃取水相, 合并有机相, 用饱和 氯化钠水溶液洗涤, 无水硫酸钠干燥, 浓缩后柱层析纯 化 $[V(\mathrm{MeOH}) / V(\mathrm{DCM})=1 / 50]$ 得到黄色油状化合物 $\mathbf{1 2}^{[7 \mathrm{~d}]}$ $179 \mathrm{mg}$, 产率 66\%. [ $\alpha]_{\mathrm{D}}^{25}-10.9$ (c 1.51, $\mathrm{CHCl}_{3}$ ); ${ }^{1} \mathrm{H}$ NMR $\left(400 \mathrm{MHz}, \mathrm{CDCl}_{3}\right) \delta: 5.08 \sim 4.97(\mathrm{~m}, 1 \mathrm{H}), 4.05 \sim$ $3.91(\mathrm{~m}, 1 \mathrm{H}), 3.65 \sim 3.28(\mathrm{~m}, 4 \mathrm{H}), 3.17 \sim 2.86(\mathrm{~m}, 2 \mathrm{H})$, $2.72(\mathrm{~s}, 1.7 \mathrm{H}$, major isomer), $2.71(\mathrm{~s}, 1.3 \mathrm{H}$, minor isomer), $2.24 \sim 1.94(\mathrm{~m}, 1 \mathrm{H}), 1.85 \sim 1.56(\mathrm{~m}, 1 \mathrm{H}), 1.47(\mathrm{~s}, 3.8 \mathrm{H}$, minor isomer), $1.46(\mathrm{~s}, 5.3 \mathrm{H}$, major isomer), $1.24(\mathrm{~s}, 5.3 \mathrm{H}$, major isomer), $1.22(\mathrm{~s}, 3.8 \mathrm{H}$, minor isomer), $0.90(\mathrm{~s}, 3.8 \mathrm{H}$, minor isomer), $0.89(\mathrm{~s}, 5.3 \mathrm{H}$, major isomer), $0.10(\mathrm{~s}, 3.4 \mathrm{H}$, major isomer), $0.08(\mathrm{~s}, 2.5 \mathrm{H}$, minor isomer); The ratio of rotamers is $1.3: 1$; ESI-LR $m / z: 549.2[\mathrm{M}+\mathrm{H}]^{+}$.

\section{辅助材料(Supporting Information) 所有合成化合物} 的核磁共振原始谱图. 这些材料可以免费从本刊网站 (http://sioc-journal.cn/)上下载.

\section{References}

[1] (a) Antibacterial agents in clinical development: an analysis of the antibacterial clinical development pipeline, including tuberculosis, World Health Organization, Geneva, 2017 (WHO/EMP/IAU/ 2017.12). License: CCBY-NC-SA 3.0 IGO.

(b) Payne, D. J.; Gwynn, M. N.; Holmes, D. J.; Pompliano, D. L. Nat. Rev. Drug Discovery 2007, 6, 29.

[2] (a) Kupferschmidt, K. Science 2016, 352, 758.

(b) Liu, Y.; Li, R.; Xiao, X.; Wang, Z. Crit. Rev. Microbiol. 2019, 45,301 .

[3] World Health Organization WHO Annual Report on Global Priority List of Antibiotic-Resistant Bacteria, 2017.

[4] (a) Hamada, M.; Takeuchi, T.; Kondo, S.; Ikeda, Y.; Naganawa. H. J. Antibiot. 1970, 23, 170. (b) Kondo, S.; Shibahara, S.; Takahashi, S.; Maeda, K.; Umezawa, H. J Am Chem Soc. 1971, 93, 6305.

[5] (a) Uehara, Y.; Hori, M.; Umezawa, H. Biochim. Biophys. Acta, Nucleic Acids Protein Synth. 1974, 374, 82.

(b) Uehara, Y.; Hori, M.; Umezawa, H. Biochim. Biophys. Acta, Nucleic Acids Protein Synth. 1976, 447, 406.

(c) Uehara, Y.; Hori, M.; Umezawa, H. Biochim. Biophys. Acta, Nucleic Acids Protein Synth. 1976, 442, 251.

(d) Mizuno, S.; Nitta, K.; Umezawa, H. J. Antibiot. 1970, 23, 589.

(e) Mizuno, S.; Nitta, K.; Umezawa, H. J. Antibiot. 1970, 23, 581.

[6] (a) Arakawa, M.; Shiozuka, M.; Nakayama Y.; Hara, T.; Hamada, M.; Kondo, A.; Ikeda, D.; Takahashi, Y.; Sawa, R.; Nonomura, Y.; Sheykholeslami, K.; Kondo, K.; Kaga, K.; Suzuki-Miyagoe, Y.; Takeda, S.; Matsuda, R. J. Biochem. 2003, 134, 751.

(b) Allamand, V.; Bidou, L.; Arakawa, M.; Floquet, C.; Shiozuka, M.; Paturneau-Jouas, M.; Gartioux, C.; Butler-Browne, G. S.; Mouly, V.; Rousset, J.-P.; Matsuda, R.; Ikeda, D.; Guicheney, P. J. Gene Med. 2008, 10, 217.

(c) Taguchi, A.; Nishiguchi, S.; Shiozuka, M.; Nomoto, T.; Ina, M.; Nojima, S.; Matsuda, R.; Nonomura, Y.; Kiso, Y.; Yamazaki, Y.; Yakushiji, F.; Hayashi, Y. ACS Med. Chem. Lett. 2012, 3, 118.

(d) Taguchi, A.; Hamada, K.; Hayashi, Y. J. Antibiot. 2018, 71, 205.

[7] Select asymmetric synthesis literature:

(a) Shibahara, S.; Kondo, S.; Maeda, K.; Umezawa, H.; Ohno, M. J. Am. Chem. Soc. 1972, 94, 4353.

(b) Masters, J. J.; Hegedus, L. S. J. Org. Chem. 1993, 58, 4547.

(c) Davies, S. G.; Ichihara, O. Tetrahedron: Asymmetry 1996, 7, 1919.

(d) Olivier, N. B.; Altman, R. B.; Noeske, J.; Basarab, G. S.; Code, E.; Ferguson, A. D.; Gao, N.; Huang, J.; Juette, M. F.; Livchak, S.; Miller, M. D.; Prince, D. B.; Cate, J. H. D.; Buurman, E. T.; Blanchard, S. C. Proc. Natl. Acad. Sci. 2014, 111, 16274.

(e) For a recent summary about the total synthesis of negamycin, see: Zhu, L.; Hong, R. Tetrahedron Lett. 2018, 59, 2112.

[8] (a) Kondo, S.; Iinuma, K.; Yoshida, K.; Yokose, K.; Ikeda, Y.; Shimazaki, M.; Umezawa, H. J. Antibiot. 1976, 29, 208.

(b) Uehara, Y.; Hori, M.; Kondo, S.; Hamada, M.; Umezawa, H. $J$. Antibiot. 1976, 29, 937.

(c) Raju, B.; Mortell, K.; Anandan, S.; O'Dowd, H.; Gao, H.; Gomez, M.; Hackbarth, C.; Wu, C.; Wang, W.; Yuan, Z.; White, R.; Trias, J.; Patel, D. V. Bioorg. Med. Chem. Lett. 2003, 13, 2413.

(d) Raju, B.; Anandan, S.; Gu, S.; Herradura, P.; O'Dowd, H.; Kim, B.; Gomez, M.; Hackbarth, C.; Wu, C.; Wang, W.; Yuan, Z.; White, R.; Trias, J.; Patel, D. V. Bioorg. Med. Chem. Lett. 2004, 14, 3103. (e) McKinney, D. C.; Basarab, G. S.; Cocozaki, A. I.; Foulk, M. A.; Miller, M. D.; Ruvinsky, A. M.; Scott, C. W.; Thakur, K.; Zhao, L.; Buurman, E. T.; Narayan, S. ACS Med. Chem. Lett. 2015, 6, 930. (f) Schmidt, U.; Stäbler, F.; Lieberknecht, A. Synthesis 1992, 482.

[9] Shibahara, S.; Kondo, S.; Maeda, K.; Umezawa, H.; Ohno, M. J. Am. Chem. Soc. 1972, 94, 4353.

[10] Liu, G.; Cogan, D. A.; Owens, T. D.; Tang, T. P.; Ellman, J. A. J. Org. Chem. 1999, 64, 1278.

[11] Ma, H. M.; Yang, L. L.; Ni, Y.; Zhang, J.; Li, C. X.; Zheng, G. W.; Yang, H. Y.; Xu, J. H. Adv. Synth. Catal. 2012, 354, 1765.

[12] (a) Reetz, M. T.; Li, X. G. J. Am. Chem. Soc. 2006, 128, 1044. (b) Ohkuma, T.; Tsutsumi, K.; Utsumi, N.; Arai, N.; Noyori, R.; Murata, K. Org. Lett. 2007, 9: 255.

[13] (a) Xu, J.; Lin, X. I. Chin. J. Org. Chem. 2007, 27, 1473 (in Chinese).

(许建明, 林贤福, 有机化学, 2007, 27, 1473.)

(b) Liao, X.; Jiang, Y.; Lai, S.; Liu, Y.; Wang, S.; Xiong, X. Chin. J. 
Org. Chem. 2019, 39, 668 (in Chinese).

(廖旭, 蒋岩, 赖石林, 刘源岗, 王士斌, 熊兴泉, 有机化学, 2019, 39, 668.)

[14] Müller, M. Angew. Chem., Int. Ed. 2005, 44, 362.

[15] Liu, G.; Cogan, D. A.; Owens, T. D.; Tang, T. P.; Ellman, J. A. J. Org. Chem. 1999, 64, 1278.
[16] (a) Tang, T. P.; Ellman, J. A. J. Org. Chem. 2002, 67, 7819.

(b) Siegel C.; Thornton, E. R. J. Am. Chem. Soc. 1989, 111, 5722

(c) Robak, M. T.; Herbage, M. A.; Ellman, J. A. Chem. Rev. 2010 , 110,3600 .

[17] Makino, K.; Jiang, H.; Suzuki, T.; Hamada, H. Tetrahedron: Asymmetry 2006, 17, 1644.

(Li, L.; Fan, Y.) 